\title{
On the Behavior of Combination High-Order Compact Approximations with Preconditioned Methods in the Diffusion-Convection Equation
}

\author{
Ahmad Golbabai ${ }^{*}$, Mahboubeh Molavi-Arabshahi ${ }^{2}$ \\ ${ }^{1}$ Islamic Azad University, Karaj Branch, Karaj, Iran \\ ${ }^{2}$ Faculty of Mathematics, Iran University of Science and Technology, Narmak, Iran \\ E-mail: $\left\{{ }^{*}\right.$ golbabai, molavi\}@iust.ac.ir \\ Received July 20, 2011; revised November 20, 2011; accepted November 24, 2011
}

\begin{abstract}
In this paper, a family of high-order compact finite difference methods in combination preconditioned methods are used for solution of the Diffusion-Convection equation. We developed numerical methods by replacing the time and space derivatives by compact finite- difference approximations. The system of resulting nonlinear finite difference equations are solved by preconditioned Krylov subspace methods. Numerical results are given to verify the behavior of high-order compact approximations in combination preconditioned methods for stability, convergence. Also, the accuracy and efficiency of the proposed scheme are considered.
\end{abstract}

Keywords: Compact High-Order Approximation, Diffusion-Convection Equation, Krylov Subspace Methods, Preconditioner

\section{Introduction}

Recently, various powerful mathematical methods such as the homotopy perturbation method, variational iteration method, Adomian decomposition method and others [1-3] have been proposed to obtain approximate solutions in partial differential equations (PDEs). The 2-D parabolic differential equations appeared in many scientific fields of engineering and science such as neutron diffusion, heat transfer and fluid flow problems. Many computational models give rise to large sparse linear systems. For such systems iterative methods are usually preferred to direct methods which are expensive both in memory and computing requirements. Krylov subspace methods are one of the widely used and successful classes of numerical algorithms for solving large and sparse systems of algebraic equations but the speed of these methods are slow for problems which arise from typical applications. In order to be effective and obtaining faster convergence, these methods should be combined with a suitable preconditioner. The convergence rate generally depends on the condition number of the corresponding matrix. Since the preconditioner plays a critical role in preconditioned Krylov subspace methods, many preconditioner have been proposed and studied [4-6]. The ADI method is a preconditioner [7,8] that can be effective for the 2-D problems but this method is not effective for more general tri-block diagonal systems. Bhuruth and Evans [9] proposed BLAGE method as a preconditioner for a class of non-symmetric linear systems. Based on author's observations, there is not a comprehensive study for comparison of preconditioning techniques to solve linear systems. In this paper, we accomplish a comprehensive study for different preconditioners in combination with Krylov subspace methods for solving linear systems arising from the compact finite difference schemes $[10$, 11] for 2-D parabolic equation

$$
\alpha u_{x x}+\beta u_{y y}=f\left(x, y, t, u, u_{x}, u_{y}, u_{t}\right)
$$

is defined in the region $W=\{x \mid 0<x, y<1, t \succ 0\}$, where $\alpha, \beta$ are positive constants. The initial conditions are:

$$
u(x, y, 0)=u_{0}(x, y), \quad 0 \leq x, y \leq 1,
$$

and boundary conditions consists of

$$
\begin{gathered}
u(0, y, t)=h_{0}(y, t), \quad u(1, y, t)=h_{1}(y, t), \quad t \succ 0 \\
u(x, 0, t)=g_{0}(x, t), \quad u(x, 1, t)=g_{1}(x, t), \quad t \succ 0,
\end{gathered}
$$

The resulting block tri-diagonal linear system of equations is solved by using Krylov subspace methods. 
Krylov subspace methods are one of the widely used and successful classes of numerical algorithms for solving large and sparse systems of algebraic equations but the speed of these methods are slow for problems which arise from typical applications [12-15]. In order to be effective and obtaining faster convergence, these methods should be combined with a suitable preconditioner. The rate of convergence generally depends on the condition number of the corresponding matrix. Since the preconditioner plays a critical role in preconditioned Krylov subspace methods, many preconditioners have been proposed and studied [6,16-18] amongst the ADI preconditioner.

In this paper, we accomplish a comprehensive study for different preconditioners in combination with Krylov subspace methods for solving linear systems arising from the compact high-order approximations. The resulting block tri-diagonal linear system of equations is solved by using Krylov subspace methods.

The outline of the paper is as follows:

In Section 2, we briefly introduce Krylov subspace methods and in Section 3, we consider some available preconditioners. In Section 4, we consider DiffusionConvection problem arising from the compact high-order approximations. We present the results of our comparative study in the final section.

\section{Krylov Subspace Methods}

Consider the linear system

$$
A x=b,
$$

where $A$ is a large sparse non-symmetric matrix. Let $x_{0}$ present an arbitrary initial guess to $x$ and $r_{0}=b-A x_{0}$ be a corresponding residual vector. An iterative scheme for solving (2.1) is called a Krylov subspace method if for any choice of $\mathrm{w}$, it produces approximate solutions of the form $x=x_{0}+w$.

In Section 4, we solve our problem with well-known Krylov subspace methods such as Generalized minimal residual method GMRES (m), Quasi minimal residual method (QMR), Bi-Conjugate Gradient method (BiCG), Conjugate gradient squared method (CGS) and Bi-Conjugate Gradient Stabilized method (BiCGSTAB) for more complete explanation refer to [13-15].

\section{Preconditioner}

The convergence rate of iterative methods highly depends on the eigen-value distribution of the coefficient matrix. A criterion for the width of the spectrum is the Euclidean condition number for SPD matrices is

$$
K=\|A\|_{2}\left\|A^{-1}\right\|_{2} \approx \lambda_{\text {max }}(A) / \lambda_{\text {min }}(A)
$$

with $\gamma=(\sqrt{K}-1)(\sqrt{K}+1)$, the distance to the exact solution $x^{*}$ in the $i^{\text {th }}$ iteration is bounded by

$$
\left\|x^{i}-x^{*}\right\|_{2} \leq 2 \sqrt{K} \gamma^{i}\left\|x^{0}-x^{*}\right\|_{2},
$$

the right hand side of (3.2) increases with growing condition number. Hence, lower condition numbers usually accelerate the speed of convergence. Hence we will attempt to transform the linear system into another equivalent system in the sense that it has the same solution, but has more favorable spectral properties. A preconditioner is a matrix that effects such as a transformation. If the preconditioner be as $M=M_{1} M_{2}$ then the preconditioned system is as

$$
M_{1}^{-1} A M_{2}^{-1}\left(M_{2} x\right)=M_{1}^{-1} b,
$$

the matrices $M_{1}$ and $M_{2}$ are called the left and right preconditioners, respectively. Now, we briefly describe preconditioners that we use for solving linear systems and matrix $A$ is block tri-diagonal.

\subsection{Preconditioner Based on Relaxation Technique}

Let $A=D+L+U$ such that $D, L$ and $U$ are diagonal, lower and upper triangular block matrices, respectively. A splitting of the coefficient matrix is as $A=M-N$ where the stationary iteration for solving a linear system is as

$$
x_{k+1}=M^{-1} N x_{k}+M^{-1} b .
$$

If the preconditioner $M$ is defined as $M=D$, then this preconditioner is called Jacobi. Also, if $M$ is defined as $M=\frac{1}{\omega}(D+\omega L)$ then we have SOR preconditioner where for $\omega=1$, we have Gauss-Seidel preconditioner. If $M$ is defined as $M=\frac{1}{\omega(2-\omega)}(D+\omega L) D^{-1}(D+\omega U)$, we get SSOR preconditioner. In the above notation, $\omega$ is called the relaxation parameter. We have chosen matrix $M$ in Jacobi, G-S and SOR methods as a left preconditioner and in SSOR preconditioner, we have chosen $M_{1}=\frac{1}{\omega(2-\omega)}(D+\omega L)$ as a left preconditioner and $M_{2}=D^{-1}(D+\omega U)$ as a right preconditioner. Also, we take $\omega_{\text {opt }}=\frac{2}{1+\sqrt{1-\rho_{J}^{2}}}[6]$.

\subsection{ADI Preconditioner}

Let $A=H+V$ and matrix $A$ is in the form 


$$
A=\left(\begin{array}{ccccc}
B_{1} & C_{1} & & & \\
A_{2} & B_{2} & C_{2} & & \\
& \ddots & \ddots & \ddots & \\
& & A_{n-1} & B_{n-1} & C_{n-1} \\
& & & A_{n} & B_{n}
\end{array}\right)
$$

where

$A_{i}=\operatorname{tridiag}\left\{a_{1 i}, b_{1 i}, c_{1 i}\right\}, B_{i}=\operatorname{tridiag}\left\{a_{2 i}, b_{2 i}, c_{2 i}\right\}$ and $C_{i}=\operatorname{tridiag}\left\{a_{3 i}, b_{3 i}, c_{3 i}\right\}$ of order $N \times N$ where $H$ and $V$ are given in the form $H=\left\{0.5 B_{i}, b_{3 i}, b_{1 i}\right\}$,

$V=\left\{0.5 B_{i}, a_{1 i}, c_{1 i}, a_{3 i}, c_{3 i}\right\}$. The alternative direction implicit method [19] for solving the linear system $A x=b$ is in following form:

$$
\begin{gathered}
\left(H+r_{1} I\right) u^{(k+1 / 2)}=b-\left(V-r_{1} I\right) u^{(k)}, \\
\left(V+r_{2} I\right) u^{(k+1)}=b-\left(H-r_{2} I\right) u^{(k+1 / 2)},
\end{gathered}
$$

The ADI preconditioner is defined as $M=\left(H+r_{1} I\right)\left(V+r_{2} I\right)$ and $M_{1}=\left(H+r_{1} I\right)$ and $M_{2}=\left(V+r_{2} I\right)$ where Parameters $r_{1}$ and $r_{2}$ are acceleration parameters. Young and Varga [20,21] proved that the optimum value for $r_{1}$ and $r_{2}$ is $\sqrt{\alpha \beta}$ where $\alpha \leq \mu_{\mathrm{i}}, v_{\mathrm{i}} \leq \beta$ and $\mu_{i}, v_{i}$ are eigen-values of matrices $\mathrm{H}$ and $\mathrm{V}$ respectively.

\subsection{BLAGE Preconditioner}

The block alternating group explicit (BLAGE) method $[22,23]$ was originally introduced as analogue of the alternating group explicit (AGE) method [24]. The BLAGE uses fractional splitting technique that is applied in two half steps on linear systems with block tri-diagonal matrices of order $N^{2} \times N^{2}$ and in the form

$$
A=\left(\begin{array}{ccccc}
B_{1} & C_{1} & & & \\
A_{2} & B_{2} & C_{2} & & \\
& \ddots & \ddots & \ddots & \\
& & A_{n-1} & B_{n-1} & C_{n-1} \\
& & & A_{n} & B_{n}
\end{array}\right)
$$

where $A_{i}, B_{i}$ and $C_{i}$ are tri-diagonal matrices of order $N \times N$. The splitting of matrix $\boldsymbol{A}$ is sum of matrices $G_{1}$ and $G_{2}$ in which $A=G_{1}+G_{2}$ where $G_{1}$ and $G_{2}$ are of the form

and

$$
G_{1}=\left(\begin{array}{cccccc}
B_{1}^{\prime} & & & & & \\
& B_{2}^{\prime} & C_{2} & & & \\
& A_{3} & B_{3}^{\prime} & & & \\
& & & \ddots & & \\
& & & & B_{n-1}^{\prime} & C_{n-1} \\
& & & & A_{n} & B_{n}^{\prime}
\end{array}\right)
$$

$$
G_{2}=\left(\begin{array}{cccccc}
B_{1}^{\prime} & C_{1} & & & & \\
A_{2} & B_{2}^{\prime} & & & & \\
& & \ddots & & & \\
& & & B_{n-2}^{\prime} & C_{n-2} & \\
& & & A_{n-1} & B_{n-1}^{\prime} & \\
& & & & & B_{n}^{\prime}
\end{array}\right)
$$

for odd values of n where $B_{i}^{\prime}=\frac{1}{2} B_{i}$. The BLAGE preconditioner is as $M=\left(G_{1}+\omega_{1} I\right)\left(G_{2}+\omega_{2} I\right)$ that $M_{1}=\left(G_{1}+\omega_{1} I\right)$ and $M_{2}=\left(G_{2}+\omega_{2} I\right)$ where $\omega_{1}$ and $\omega_{2}$ are optimal iteration parameters. We have experimentally chosen the relaxation parameter $\omega_{1}=\sqrt{\alpha_{1} \beta_{2}}$ and $\omega_{2}=\sqrt{\alpha_{2} \beta_{1}}$ where $\alpha_{1}=\lambda_{\text {min }}\left(M_{1}\right)$, $\beta_{1}=\lambda_{\text {max }}\left(M_{1}\right)$ and $\alpha_{2}=\lambda_{\text {min }}\left(M_{2}\right), \quad \beta_{2}=\lambda_{\text {max }}\left(M_{2}\right)$ so that we will have the minimum condition number.

\section{Numerical Illustrations}

In this section, we present one numerical example to show the computational efficiency of the preconditioner which introduced in Section 3. Our initial guess is the zero vector and the iterations are stopped when the relative residual is less than $10^{-6}$. We show the number of outer iterations and inner iterations GMRES (m) method with "ou" and "in" respectively in following tables. Also, we show the iteration number without using preconditioner by "no pre" and the coefficient matrix is order of $N^{2} \times N^{2}$. The computations have been done on a P.C. with Corw 2 Pue 2.0 Ghz and 1024 MB RAM.

Test: We consider 2-D partial differential equation:

$$
\alpha u_{x x}+\beta u_{y y}=u_{x}+u_{y}+r u_{t}-\exp (-t) \cos (x+y)
$$

with Dirichlet boundary conditions on the unit square where

$$
u(x, y, t)=\exp (-t) \cos (x) \sin (y) .
$$

where $\alpha=\beta=5 \times 10^{-4}$. We apply the fourth-order approximation for discretization of Equation (4.1) (Figure 1). We have shown the number of iteration for different preconditioned methods in Tables 1-5 or different preconditioners. The convergence behavior of preconditioned Krylov subspace methods is given by Figures 2-7. Also, in Figures 8-10 for we show the distribution of eigen-values SSOR, ADI and BLAGE preconditioners. In this test the condition number of problem is high and our problem is ill-conditioned. So, in regard of other preconditioner, results show the ADI preconditioner requires more iteration. It is seen that we obtain the optimal convergence with SSOR and BLAGE preconditioner and our time consumption is reduced. 
Tabale 1. Number of iterations with GMRES.

\begin{tabular}{ccccccc}
\hline $\mathrm{h}$ & no pre & Jacobi & SOR & SSOR & ADI & BLAGE \\
\hline $1 / 20$ & 75 & 54 & 36 & 21 & 33 & 26 \\
$1 / 40$ & 124 & 89 & 61 & 36 & 61 & 51 \\
$1 / 60$ & 119 & 87 & 60 & 35 & 65 & 54 \\
$1 / 80$ & 139 & 102 & 58 & 37 & 76 & 63 \\
$1 / 100$ & 162 & 119 & 58 & 40 & 87 & 72 \\
\hline
\end{tabular}

Tabale 2. Number of iterations with QMR.

\begin{tabular}{ccccccc}
\hline $\mathrm{h}$ & no pre & Jacobi & SOR & SSOR & ADI & BLAGE \\
\hline $1 / 20$ & 79 & 56 & 48 & 22 & 40 & 34 \\
$1 / 40$ & 152 & 96 & 99 & 33 & 72 & 55 \\
$1 / 60$ & 162 & 109 & 95 & 39 & 77 & 61 \\
$1 / 80$ & 189 & 137 & 67 & 34 & 97 & 77 \\
$1 / 100$ & 225 & 149 & 63 & 39 & 102 & 79 \\
\hline
\end{tabular}

Tabale 3. Number of iterations with CGS.

\begin{tabular}{ccccccc}
\hline $\mathrm{H}$ & no pre & Jacobi & SOR & SSOR & ADI & BLAGE \\
\hline $1 / 20$ & 62 & 36 & 28 & 14 & 27 & 18 \\
$1 / 40$ & 81 & 50 & 43 & 18 & 40 & 35 \\
$1 / 60$ & 93 & 56 & 37 & 20 & 42 & 34 \\
$1 / 80$ & 114 & 74 & 41 & 26 & 57 & 47 \\
$1 / 100$ & 146 & $\mathrm{~N}$ & 42 & 31 & 73 & 49 \\
\hline
\end{tabular}

Tabale 4. Number of iterations with BiCG.

\begin{tabular}{ccccccc}
\hline $\mathrm{h}$ & no pre & Jacobi & SOR & SSOR & ADI & BLAGE \\
\hline $1 / 20$ & 85 & 57 & 52 & 22 & 41 & 34 \\
$1 / 40$ & 152 & 99 & 97 & 36 & 72 & 55 \\
$1 / 60$ & 162 & 108 & 86 & 39 & 77 & 67 \\
$1 / 80$ & 190 & 137 & 67 & 34 & 95 & 73 \\
$1 / 100$ & 223 & 131 & 67 & 39 & 99 & 87 \\
\hline
\end{tabular}

Tabale 5. Number of iterations with BiCGSTAB.

\begin{tabular}{ccccccc}
\hline $\mathrm{h}$ & no pre & Jacobi & SOR & SSOR & ADI & BLAGE \\
\hline $1 / 20$ & 62 & 46 & 23 & 15 & 31 & 17 \\
$1 / 40$ & 81 & 60 & 36 & 21 & 43 & 25 \\
$1 / 60$ & 86 & 54 & 38 & 19 & 45 & 30 \\
$1 / 80$ & 103 & 72 & 37 & 22 & 52 & 41 \\
$1 / 100$ & 135 & 95 & 36 & 26 & 65 & 49 \\
\hline
\end{tabular}

Convection-diffusion problem

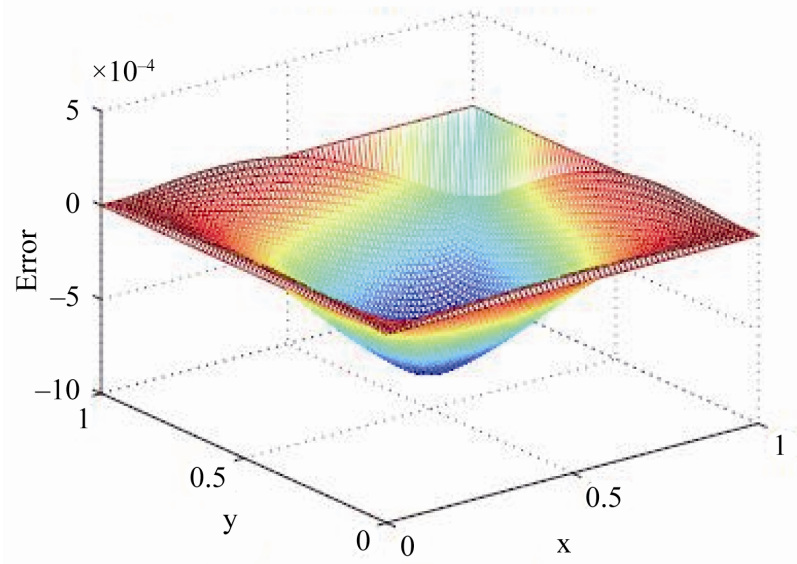

Figure 1. The 3D error of the compact fite diffence scheme with time $T=10$.

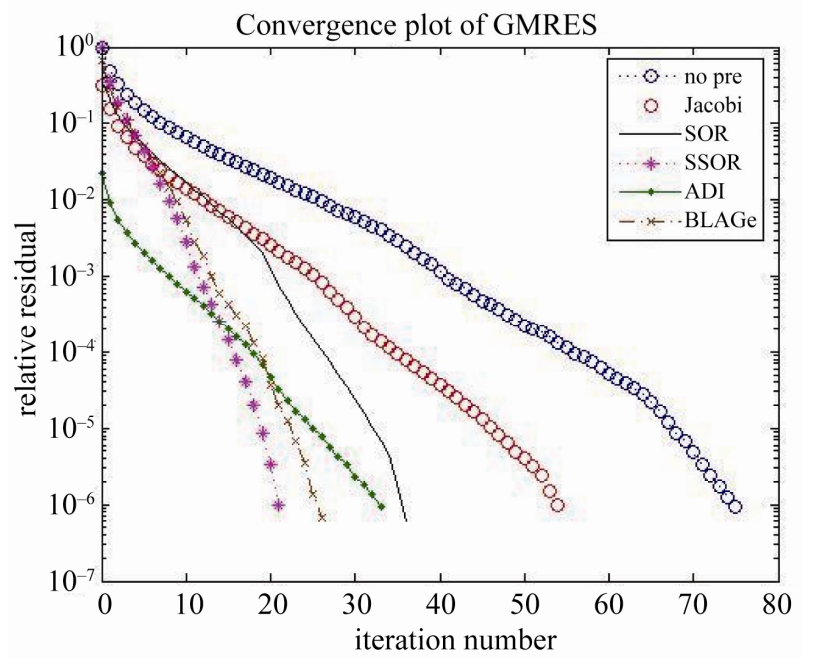

Figure 2. Convergence plot of GMRES.

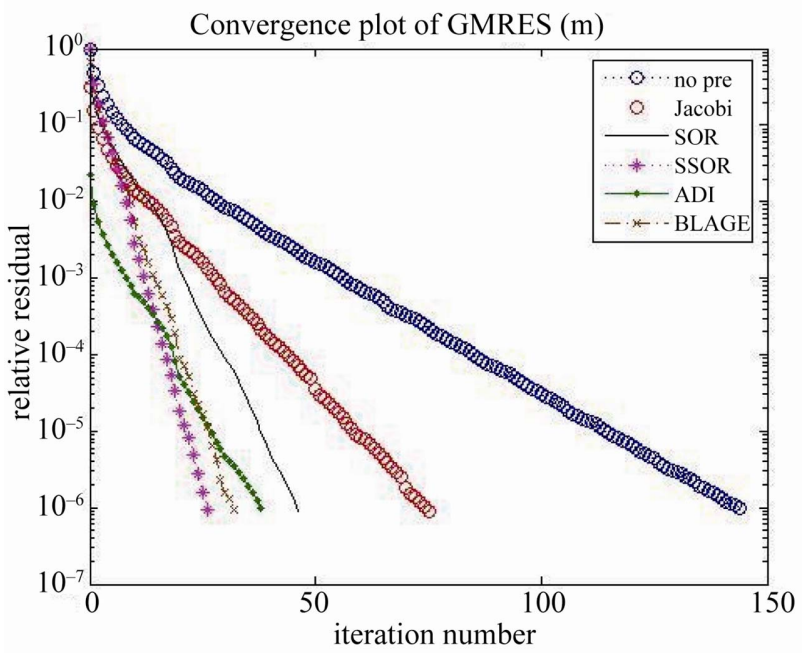

Figure 3. Convergence plot of GMRES (15). 


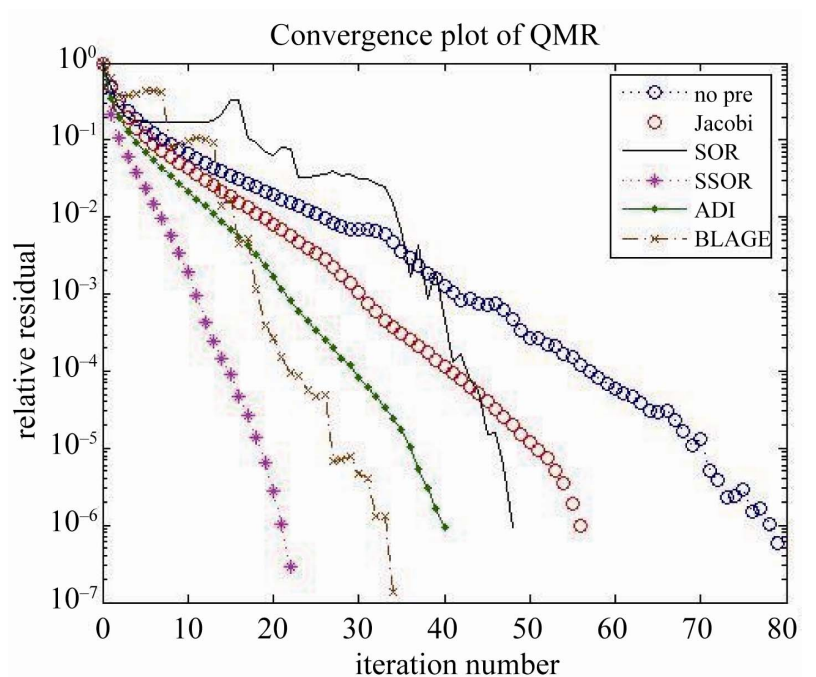

Figure 4. Convergence plot of QMR.

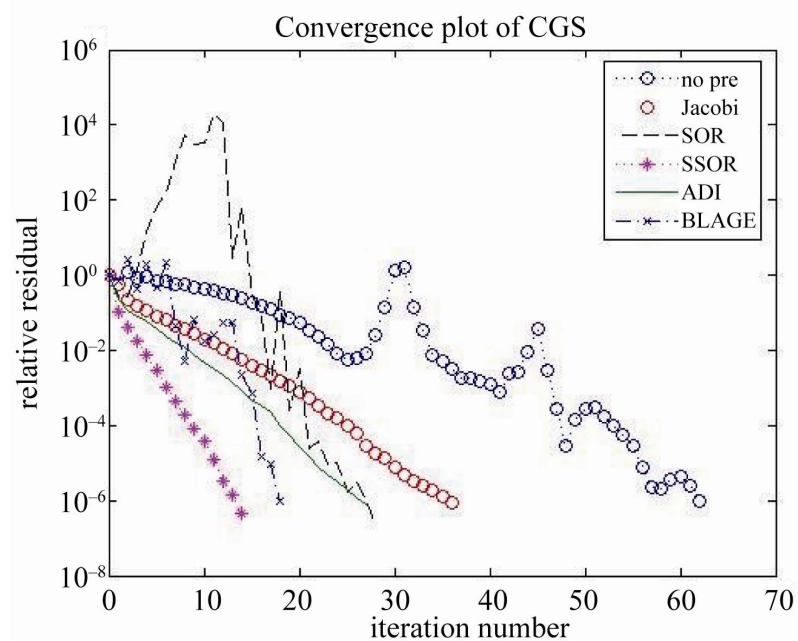

Figure 5. Convergence plot of CGS.

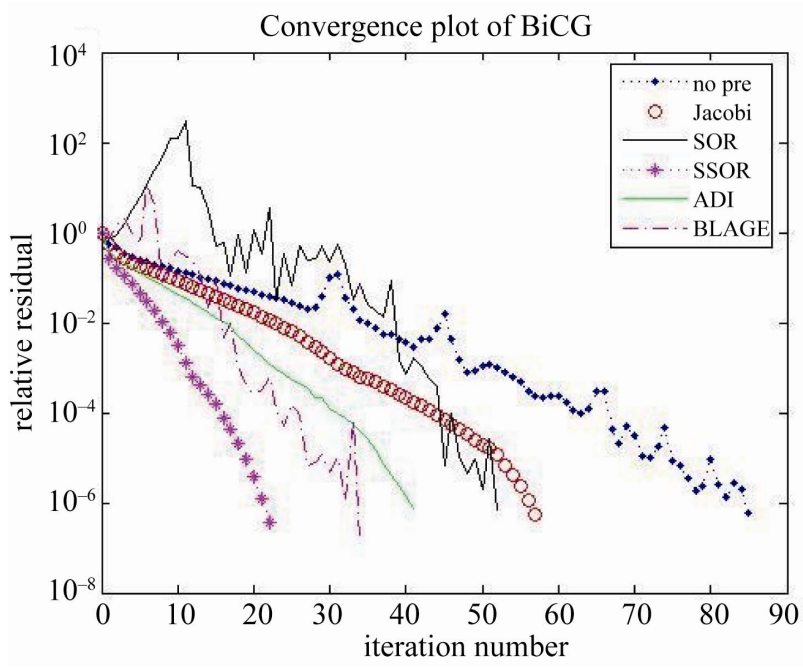

Figure 6. Convergence plot of BiCG.

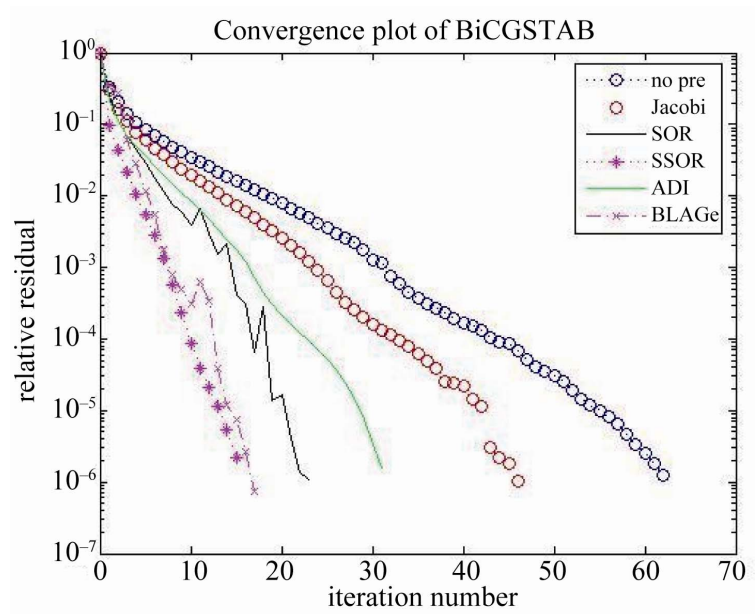

Figure 7. Convergence plot of BiCGSTAB.
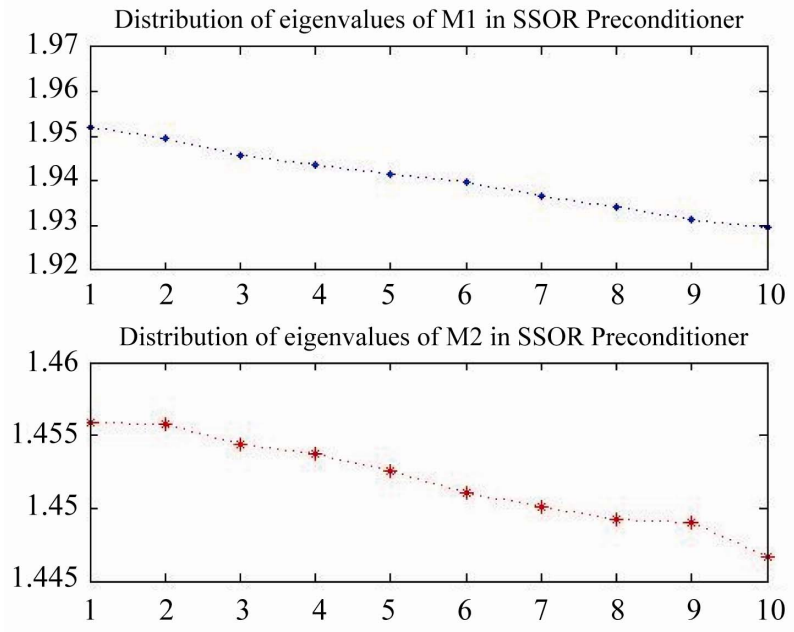

Figure 8. Distribution of eigen-values in SSOR preconditioner.
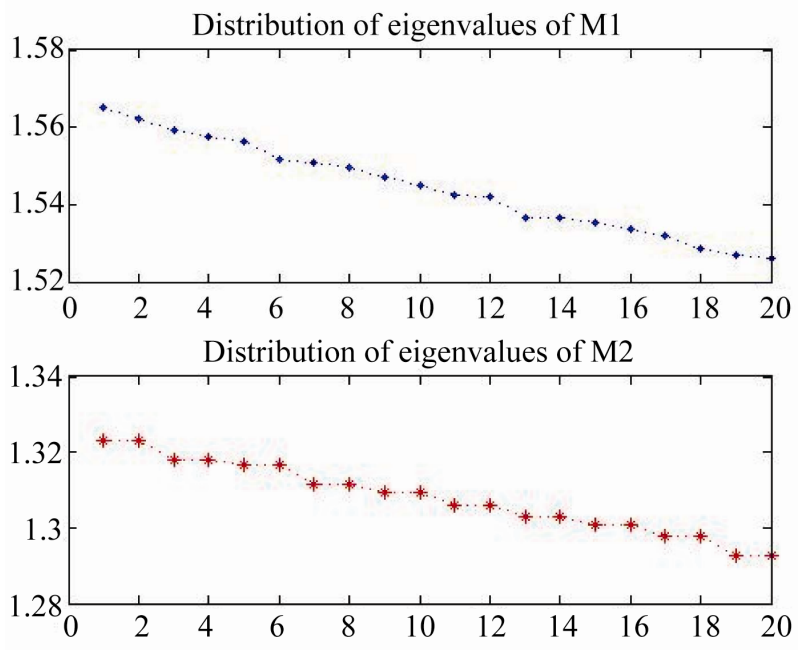

Figure 9. Distribution of eigen-values in ADI Preconditioner. 

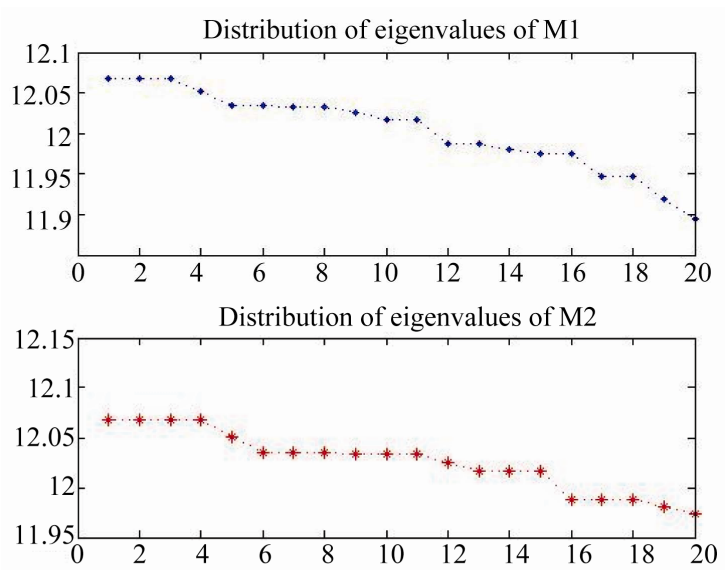

Figure 10. Distribution of eigen-values in BLAGE preconditioner.

\section{Conclusions}

A high-order compact scheme in combination preconditioner was applied successfully to Diffusion- Convection equation. We study comparison of different preconditioners in combination Krylov subspace methods. Highorder approximation are designed by the need to produce more stable schemes which are efficient with respect to the operation number and that do not experience difficulties near boundaries. The numerical results which is given in the previous section demonstrate the good accuracy of this scheme and efficiency of preconditioned Krylov subspace methods. We got to this conclusion that the ADI preconditioner is effective for model problems rather than other. So we propose using ADI preconditioner in combination with Krylov subspace methods for solving non-symmetric systems because this preconditioner needs to less computing time and have the less iteration number than other. Also, we propose the BiCGSTAB method because of the need to less iteration number, simplicity in implementation, flat convergence and to save in comp tational time.

\section{References}

[1] L. C. Evans, "Partial Differential Equations," American Mathematical Society Providence, Rhode Island, 1999.

[2] A. Golbabai and M. M. Arabshahi, “A Numerical Method for Diffusion-Convection Equation Using High-Order Difference Schemes," Computer Physics Communications, Vol. 181, No. 7, 2010, pp. 1224-1230. doi:10.1016/j.cpc.2010.03.008

[3] A. Golbabai and M. M. Arabshahi, "On the Behavior of High-Order Compact Approximations in One Dimensional Sine-Gordon Equation,” Physica Scripta, Vol. 83, No. 1, 2011, Article ID 015015. doi:10.1088/0031-8949/83/01/015015
[4] L. C. Evans, "Partial Differential Equations,” American Mathematical Society Providence, Rhode Island, 1999.

[5] S. Sundar and B. K. Bhagavan, "CGS, Comparison of Krylov Subspace Methods with Preconditioning Techniques for Solving Boundary Value Problems," Computers and Mathematics with Applications, Vol. 38, No. 11-12, 1999, pp. 197-206. doi:10.1016/S0898-1221(99)00298-9

[6] A. M. Bruaset, “A Survey of Preconditioned Iterative Methods,” Longman Scientific and Technical, UK, 1995.

[7] K. J. Hout and B. D. Welfert, "Unconditional Stability of Second-Order ADI Schemes Applied to Multi-Dimensional Diffusion Equations with Mixed Derivative Terms," Applied Numerical Mathematics, 2008, Article in Press.

[8] S. Ma and Y. Saad, "Block-ADI Preconditioners for Solving Sparse Non-Symmetric Linear Systems of Equations,” Numerical Linear Algebra, 1993, pp. 165-178.

[9] M. Bhuruth and D. J. Evans, "Block Alternating Group Explicit Preconditioning (BLAGE) for a Class of FourthOrder Difference Schemes," International Journal of Computer Mathematics, Vol. 63, No. 1-2, 1997, pp. 121136. doi:10.1080/00207169708804555

[10] M. K. Jain, R. K. Jain and R. K. Mohanty, "Fourth-Order Finite Difference Method for 2-D Parabolic Partial Differential Equations with Non-Linear First-Derivative Terms," Numerical Methods for Partial Differential Equations, Vol. 8, No. 1, 1992, pp. 21-31. doi:10.1002/num.1690080102

[11] G. I. Shishkin and L. P. Shishkina, “A Higher Order Richardson Scheme for a Singularly Perturbed Semilinear Elliptic Convection-Diffusion Equation,” Computational Mathematics and Mathematical Physics, Vol. 50, No. 3, 2010, pp. 437-456. doi:10.1134/S0965542510030061

[12] Y. Zhang, "Matrix Theory Basic Results and Techniques,” Springer, Berlin, 1999.

[13] R. Barrett, et al., "Templates for the Solution of Linear Systems: Building Blocks for Iterative Methods,” Society for Industrial and Applied Mathematics (SIAM), 1994, pp. xvii+118. doi:10.1137/1.9781611971538

[14] Y. Saad, "Iterative Methods for Sparse Linear Systems," Second Edition, PWS Publishing Company, Boston, 2000.

[15] H. A. Van der Vorst, "Iterative Krylov Subspace Methods for Large Linear Systems,” Cambridge University Press, Cambridge, 2003. doi:10.1017/CBO9780511615115

[16] O. Axelsson, "Iterative Solution Methods," Cambridge University Press, New York, 1996.

[17] M. H. Koulaei and F. Toutounian, “On Computing of Block ILU Preconditioner for Block Tri-Diagonal Systems," Journal of Computational and Applied Mathematics, Vol. 202, No. 2, 2007, pp. 248-257. doi:10.1016/j.cam.2006.02.029

[18] R.C. Mittal and A.H. Al-Kurdi, "An Efficient Method for Constructing an ILU Preconditioner for Solving Large Sparse Non-Symmetric Linear Systems by the GMRES Method," Computers and Mathematics with Applications, Vol. 45, 2003, pp. 1757-1772. 
doi:10.1016/S0898-1221(03)00154-8

[19] D. W. Peaceman and H. H. Rachford, "The Numerical Solution of Parabolic and Elliptic Differential Equations," Journal of the Society for Industrial and Applied Mathematics, Vol. 3, No. 1, 1955, pp. 28-41. doi: $10.1137 / 0103003$

[20] D. M. Young, "Iterative Solution of Large Linear Systems,” Academic Press, New York, 1971.

[21] R. S. Varga, "Matrix Iterative Analysis,” Prentice Hall, Englewood Cliffs, 1962.

[22] D. J. Evans and W. S. Yousif, "The Block Alternating Group Explicit Method (BLAGE) for the Solution of Elliptic Difference Equations," International Journal of
Computer Mathematics, Vol. 22, No. 2, 1987, pp. $177-$ 185. doi:10.1080/00207168708803590

[23] R. K. Mohanty, “Three-Step BLAGE Iterative Method for Two-Dimensional Elliptic Boundary Value Problems with Singularity," International Journal of Computer Mathematics, Vol. 84, No. 11, 2007, pp. 1613-1624. doi:10.1080/00207160600825205

[24] D. J. Evans and M. Sahimi, "The Alternating Group Explicit (AGE) Iterative Method to Solve Parabolic and Hyperbolic Partial Differential Equations,” Annual Review of Numerical Fluid Mechanics and Heat Transfer, Vol. 11, 1989, pp. 283-390. 